\title{
Residual Motion Perception in a "Motion-Blind" Patient, Assessed with Limited-Lifetime Random Dot Stimuli
}

\author{
Curtis L. Baker, Jr., ${ }^{1}$ Robert F. Hess, ${ }^{2}$ and Josef Zihl ${ }^{3}$ \\ 'Department of Psychology and ${ }^{2}$ McGill Vision Research Unit, Department of Ophthalmology, McGill University, Montreal, \\ Quebec, Canada H3A 1B1, and Max-Planck-Institut fur Psychiatrie, 8000 Munchen 40, Federal Republic of Germany
}

\begin{abstract}
A neurological patient (L.M.) suffering a specific loss of visual motion perception (Zihl et al., 1983) due to extrastriate cortical damage was studied using random dot "limited-lifetime" stimuli with a direction discrimination task. With a stimulus like that of Newsome and Pare (1988), the patient exhibited a severe deficit for motion perception, only being able to perform well for very high values of coherence.

Different versions of the stimulus were employed to separate out the effects of limited lifetime versus the effects of additive noise as coherence was lowered. When all "signal" dots had a fixed, specified value of lifetime, and varying percentages of "noise" dots were added, the patient showed a profound deficit. In contrast, a stimulus consisting of no noise dots at all, and signal dots having fixed values of lifetime, revealed relatively good performance for surprisingly brief dot lifetimes. Thus, it is the presence of noisy, incoherent dot motion, rather than brief lifetimes, that causes such poor performance on the stimulus of Newsome and Pare (1988). Most surprising was the finding that the presence of even very small percentages of stationary noise dots was sufficient to disrupt totally direction discrimination of moving signal dots.

The findings reported here suggest that one major role of extrastriate cortical processing might be the interpretation of stimuli that suffer from an impaired signal-to-noise ratio; the most commonly encountered form of "noise" would presumably be contamination by irrelevant directional spatiotemporal frequency components.
\end{abstract}

Neuroanatomical research over the past 2 decades has revealed a multiplicity of extrastriate visual cortical areas, each containing its own retinotopic map of at least part of the visual field (Allman and Kaas, 1971; Zeki, 1974; van Essen, 1985; Maunsell and Newsome, 1987). One of the major problems of modern visual neuroscience is to ascertain the functions of these areas.

One approach to this problem is to study the nature of visual

\footnotetext{
Received Mar. 16, 1990; revised Aug. 14, 1990; accepted Sept. 20, 1990

We thank Christel Schmid for her assistance in some of these experiments, Wolfram Zahn for help with the computer configuration, and Cathryn Downing and $J$. Anthony Movshon for helpful discussions and for providing us with their data before publication. We also thank Jane Boulton for comments on the manuscript. We are particularly grateful to the patient, L.M., who so generously agreed to participate in these lengthy experiments. This research was supported by $\mathrm{Ca}$ nadian NSERC Grant A1978 to C.B., a Canadian NSERC Grant to R.L.H., and the Max-Planck-Institut fur Psychiatrie. R.F.H. is a Wellcome Senior Lecturer.

Correspondence should be addressed to Curtis L. Baker, Jr., Department of Psychology, McGill University, 1205 Avenue Docteur Penfield, Montreal, Quebec, Canada H3A 1BI.

Copyright $\odot 1991$ Society for Neuroscience $0270-6474 / 91 / 110454-08 \$ 03.00 / 0$
}

perceptual losses in human patients, or animals, who have suffered selective lesions of some restricted subset of these areas. Zihl et al. (1983) described a patient having a bilateral loss in the superior temporal region, who complained of anomalous or impaired motion perception. They used extensive neuropsychological tests to document the patient's poor performance on motion-related visual tasks. Most striking was the patient's normal performance on other perceptual tasks not involving motion, for example, Snellen and vernier acuity, temporal resolution, stereopsis, color discrimination, and saccadic localization. The location of this patient's lesion was found to be in the general topographic vicinity corresponding to area MT in the monkey (Allman and Kaas, 1971; van Essen, 1985), also referred to as area V5 (Zeki, 1974). The pervasiveness of strongly direction-selective neurons (Dubner and Zeki, 1971) and the loss of performance in psychophysical tasks requiring motion perception in MT-lesioned monkeys (Newsome and Pare, 1988; Siegel and Andersen, 1988), together, suggest an important role of this area in motion perception.

Later studies on this same patient (Hess et al., 1989) used forced-choice discrimination psychophysics and sine-wave grating stimuli to document further the nature of this patient's visual loss. Contrast sensitivity for simple detection of sine-wave gratings was shown to exhibit only a very small loss, even for moving gratings; however, contrast sensitivity contingent on discrimination of direction of motion, for the same stimuli, was severely reduced. Grating spatial-frequency discrimination and contrast discrimination were somewhat impaired (again, similarly for moving and stationary stimuli), but considerably less so than temporal frequency or velocity discrimination for the same stimuli. These findings largely confirmed the specificity of the deficit to motion perception, but demonstrated that the loss was for judgment of stimulus motion attributes, rather than a reduced sensitivity to moving stimuli.

Hess et al. (1989) also tested the patient's motion perception using random dot stimuli. Somewhat surprisingly, they found that she could perform well on direction discrimination of smoothly drifting random dot patterns, albeit only over a considerably more restricted range of velocities than in the normal observer. Testing with double-flash random dot kinematograms revealed a very large deficit, but some residual performance if the cxposurc duration of the flashes was made very long. These experiments were somewhat unsatisfactory in that such longduration stimuli might provide the opportunity to make indirect inference of motion by scrutiny of dot clusters. On the other hand, the poor performance might be due, in part, to 2-flash motion stimuli containing substantial amounts of spurious spatiotemporal frequency components (some in the opposite di- 
rection of motion; scc Fig. 5 of van Santen and Sperling, 1985); perhaps her deficit involved a lack of ability to separate out these irrelevant signals. Thus, it would be desirable to disambiguate the effects of overall exposure time to the stimulus versus "noise" from undesired spatiotemporal frequency components.

To this end, we desired to use a somewhat different type of "2-flash" stimulus that could be of potentially indefinite exposure duration, yet still allow analytical manipulation of spatial displacement and temporal interval. Furthermore, it seemed desirable to have a richer measure of performance than, for instance, $\mathrm{D}_{\max }$ or $\mathrm{D}_{\min }$, but rather an indicator of the quality of perceived motion for values of displacement intermediate between these limits. This would enhance our chances of uncovering whatever residual motion perception competence that might be present. Finally, we wanted a stimulus that could provide results with potential for direct comparison with data from animal cxpcriments.

For this purpose, we chose to use a random "limited-lifetime" stimulus in the general category of those developed by Morgan and Ward (1980), Williams and Sekuler (1984), Zucker (1984), and Siegel and Andersen (1988). Initially, we used the particular implementation developed by J. A. Movshon, which was first employed by Newsome and Pare (1988). The latter authors demonstrated that a small lesion restricted to primate area MT causes a severe deficit in motion perception using this stimulus, while not causing a deficit for grating contrast sensitivity.

Here, we report the results of such experiments on the patient (now referred to as L.M.) of Zihl et al. (1983), which reveal a deficit strikingly similar to that of Newsome and Pare's (1988) MT-lesioned monkeys. In a further series of experiments, we employ various other kinds of stochastic motion stimuli in an effort to disambiguate whether the deficit is fundamentally a temporal problem (a necd for longer "intcgration timc" or "rccruitment" across multiple flashes), as opposed to a signal/noise problem; our results are indicative of the latter.

\section{Materials and Methods}

Stimulus. In the first, simplest version of the stimulus (here referred to as "Movshon noise"), we sought to produce a stimulus as close as possible to that used by Newsome and Pare (1988) and by Downing and Movshon (1989). Later experiments employed a number of variations on this theme. All of these stimuli provided motion information for direction discrimination judgments and exhibited the advantageous features of being balanced in luminance, and spatial and temporal attributes, such that the only possible basis of the observer's direction discrimination had of necessity to be spatiotemporal correlation (i.e., "motion").

The Movshon noise stimulus consisted of an on-going series of dots, sequentially plotted one every $200 \mu \mathrm{sec}$, from a precalculated list. This list contained $x$ - and $y$-coordinates of the number of dots (ndots) that could be plotted in one nominal "frame time," for the full stimulus duration of $1000 \mathrm{msec}$. The locations of the initial ndots were randomly chosen; then, for each ensuing "frame," a given dot would "live" or "die" according to a specified probability, the coherence value. A dot that lives is then replotted at the specified displacement to the left (or right) of its previous location, subject to wraparound at the edges of the display; one that dies is replotted at a freshly chosen (uncorrelated) random location. Note that a $100 \%$ value of the coherence parameter yields a smoothly drifting field of randomly positioned dots; 0 coherence results in a totally incoherent "snowstorm" devoid of net directional information. Also note that the effective interstimulus interval (ISI) between successive refreshes of a given dot is equal to the frame time.

Later versions of the stimulus ("dot noise," "immortal noise," etc.) differed principally in that the dots were explicitly divided into 2 subsets: signal dots and noise dots. In these stimuli, the coherence parameter refers to the percentage of total displayed dots that were signal dots. A given signal dot was refreshed on the screen every ISI msec, at successive displacement jumps, until the end of its lifetime, whereupon its trajectory died and was "reborn" at a fresh random display coordinate. The noise dots were replotted at fresh random coordinates on each frame refresh. Different versions of the stimulus (e.g., immortal noise, etc.) differed in minor but important ways in the rules governing dot life and death, which will be described below.

Stimuli were generated in the following manner: for each trial, lists of $x$ - and $y$-coordinates of random dots were first calculated and stored in microcomputer RAM. Upon initiation of a trial, numbers from these lists were very rapidly loaded into the 212 -bit digital-to-analog converters of a lab interface card. The resultant voltages were used to drive the $x$ and $y$ deflections of a Tektronix 613 XY display monitor (P1 phosphor). Following a suitable interval for beam-deflection settling time, the display monitor bright-up control was briefly activated through a digital output bit from the lab interface. Using this procedure with highly optimized C-language code (compiled with Microsoft $\mathrm{C}$, version 5.1 ) running on a $20-\mathrm{MHz} 80386$-based microcomputer, it was possible to display dots at a synchronously controlled rate of $5 \mathrm{kHz}$; this allowed, for example, 225 dots to be displayed in a time of $45 \mathrm{msec}$, yielding a dot density comparable to that in the display described by Newsome and Pare (1988).

The spatial configuration of our stimulus consisted of a square array of dots, $10.7^{\circ} \times 8.0^{\circ}$ of visual angle, viewed at a distance of $114 \mathrm{~cm}$ and centrally fixated.

Each trial was initiated by a button press, resulting in a single 1000 msec stimulus presentation. The observer was then required to indicate the perceived (or guessed) direction of motion, left versus right, that was registered by the microcomputer.

Room lighting was reduced relative to normal illumination, in order to maximize dot contrast. However, adequate background light was used to help minimize problems associated with stray light and with phosphor persistence on the CR'1. The background luminance on the random dot display was $0.1 \mathrm{~cd} / \mathrm{m}^{2}$, as measured with a Spectra Spotmeter. A luminance of $50 \mathrm{~cd} / \mathrm{m}^{2}$ was measured for the random dots, with the reading taken on a display consisting of dots in a regular tessellation, spaced $1 \mathrm{~min}$ arc apart.

Procedures. The procedures employed were much as in a previous paper (Hess et al., 1989). We employed 2-alternative forced choice, with a "method of constant stimuli."

As previously described (Hess et al., 1989), the patient L.M. was not required to press mouse buttons manually, but instead to indicate responses verbally or gesturally. Data were collected in very short sessions, typically consisting of 4 stimulus conditions with 20 trials per condition, presented in a randomly interleaved fashion. Sets of such data were accumulated, while providing the patient frequent intervals for rest, to help circumvent her complaint of a tendency to fatigue rapidly as a result of viewing moving stimuli.

Observers. Performance of normal observers was obtained from the authors or the lab assistant (C.S.).

Data analysis. Data from successive trial blocks were accumulated to obtain psychometric functions of percentage correct versus percentage coherence, with 40 or more trials per condition. In some cases, these data were parameterized by obtaining a least-squares fit of a cumulative function (Weibull, 1951) to extract a quantitative measure of threshold coherence corresponding to $81.6 \%$ correct. Such curve fits were performed on a log-scaled abscissa.

\section{Results}

\section{Movshon noise}

Because recent magnetic-resonance-imaging (MRI) scan data (Zihl et al., 1991) confirmed that L.M.'s lesion is bilateral, but is more pronounced in the right hemisphere, we sought to assess the impact of this asymmetry on our psychophysical tests. For this purpose, we used the Movshon noise stimulus, with a spatial displacement of $4.2 \mathrm{~min}$ arc and an ISI of $45 \mathrm{msec}$. Unlike all the other tests described here, the patient was asked to fixate $2.3^{\circ}(4.5 \mathrm{~cm}$ at a viewing distance of $114 \mathrm{~cm})$ to either the right or the left side of the edge of the display, thus confining the stimulus entirely to either the left or right hemifield. Psychometric functions of percent correct versus percent coherence from this experiment are shown in Figure 1 and indicate very 


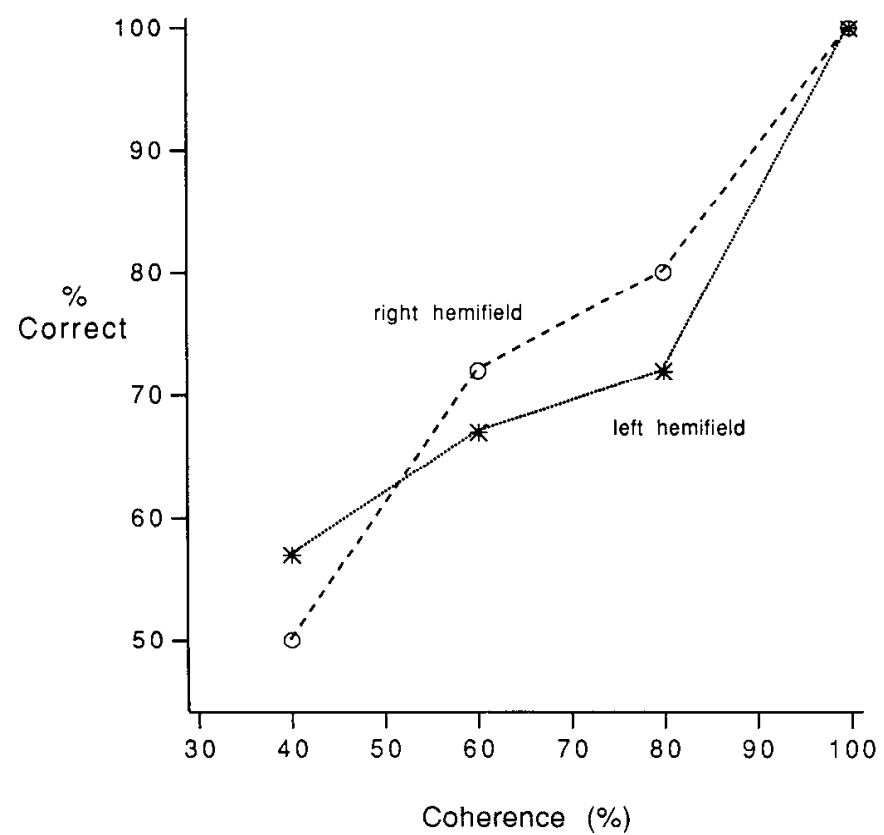

Figure 1. Psychometric functions for L.M.'s direction discrimination as function of coherence parameter, tested separately in left and right hemifields. Note that performance deteriorates with decreasing coherence, over a range in which normal observers would exhibit $100 \%$ correct performance.

little difference between the hemifields. Consequently, in all further tests, we employed central fixation of the stimulus field.

Given the degree to which many extrastriate cortical neuron receptive fields straddle the midline, this test may not definitively address the question of psychophysical correlation with the lesion asymmetry. However, it at least demonstrates that there was no (lateral) visual field inhomogeneity in performance on this task, over the region in which subsequent tests were made.

What is already quite clear in these data, however, is the patient's very poor performance; for these conditions, an observer having normal vision would perform at $100 \%$ correct for all the coherence values tested. We next set about to document the dependence of this deficit on the spatial and temporal parameters of the stochastic stimulus. For a given set of values of spatial displacement and ISI, we measured a psychometric function like that shown in Figure 1 and used a Weibull curve fit to obtain an estimate of the coherence threshold corresponding to $81.6 \%$ correct.

Figure 2 shows these threshold coherence values as a function of spatial displacement, for a fixed ISI of $45 \mathrm{msec}$. Broken lines illustrate data for 2 normal observers, who produced thresholds as low as $2-5 \%$ coherence for optimal values of spatial displacement (2-20 min arc). The patient, however, exhibited highly elevated threshold coherence values, of more than $80 \%$. At the extreme values of very small or very large displacement, at which the normal observers had elevated thrcsholds, she was unable to perform the task at all (even for $100 \%$ coherence).

Similar measurements as a function of ISI, keeping displacement fixed at $4.2 \mathrm{~min}$ arc, are shown in Figure 3. Again, normal observers produced coherence thresholds as low as $5 \%$ for optimal ISI values of about $40-80 \mathrm{msec}$, with elevated thresholds for lower or higher values, while the patient's deficit was again

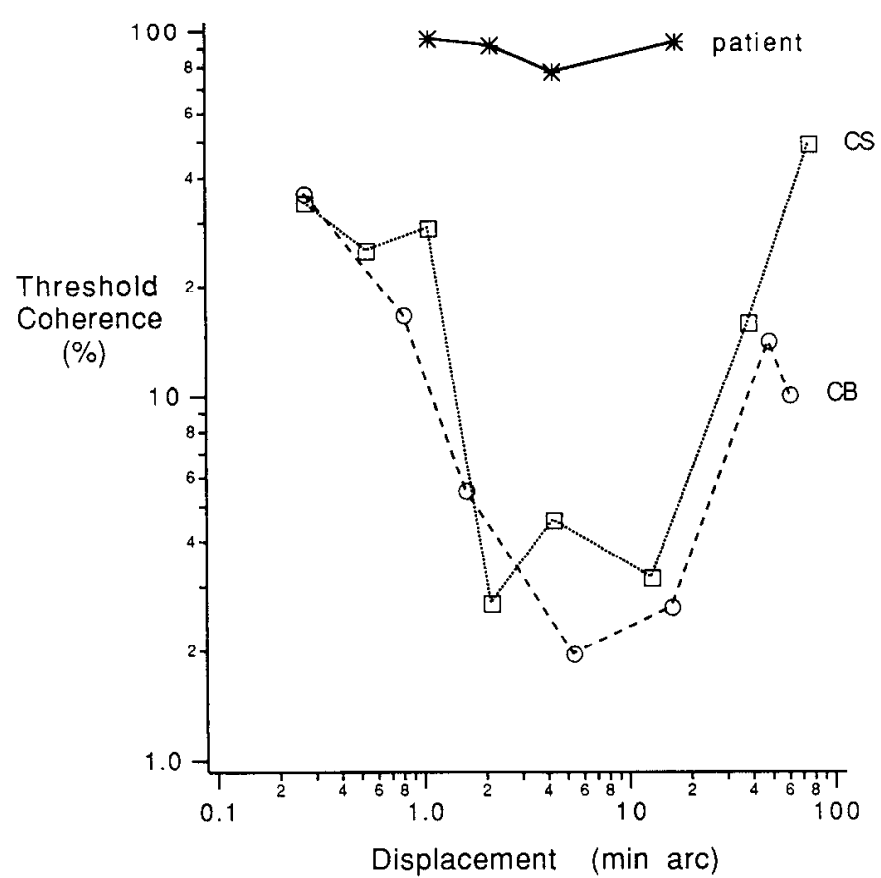

Figure 2. Threshold values of percent coherence, using Movshon noise stimulus, as function of given dot's spatial displacement on successive frames. Each plotted point represents the interpolated coherence value corresponding to $81.6 \%$ correct in a measured psychometric function like that shown in Figure 1. Normal observers $(C S, C B)$ exhibited superior performance (i.e., low thresholds of 2-5\%) over a range of displacements from 2-20 min arc, with decreased performance for either higher or lower values. The patient L.M., however, showed significant impairment throughout this range, with coherence thresholds above $80 \%$. In this and all succeeding figures, normal observers' data are indicated with open symbols and broken lines, while the patient L.M.'s data are indicated with asterisk symbols and solid lines.

very great at all ISIs. She was unable to perform the task at all for ISI values higher than about $100 \mathrm{msec}$.

These data confirmed our previous finding (Hess et al., 1989) that L.M. could reliably discriminate direction of motion for drifting random dot patterns (100\% cohcrence). However, the introduction of even very small degrees of noncoherence resulted in a rapid deterioration of performance. This decline in performance correlated with a seemingly qualitative shift in the perceptual appearance of these stimuli, from a smoothly drifting, rigid sheet of random dots (at 100\% coherence) to a noisy, flickering field of dots, each having a very limited lifetime (at, e.g., $70 \%$ coherence). The question arose as to whether L.M.'s poor performance was due to the introduction of noise or, alternatively, to the reduction of dot lifetime that is concomitant with reduced coherence. The experiments described below were designed to disambiguate these possibilities.

\section{Dot noise}

In these experiments, we used a modified version of the stimulus, in which dots were divided into 2 subsets: signal dots and noisc dots. Signal dots cach jumped by a specified displacement every $45 \mathrm{msec}$, for a specified number of jumps (the dot lifetime); thereupon, the dot would die and be reborn at a randomly determined new location $45 \mathrm{msec}$ later, then embark on a new trajectory of the same specified lifetime. In the course of their lifetimes, all signal dots moved in the same direction, left or right. Noise dots, however, jumped to new random locations 


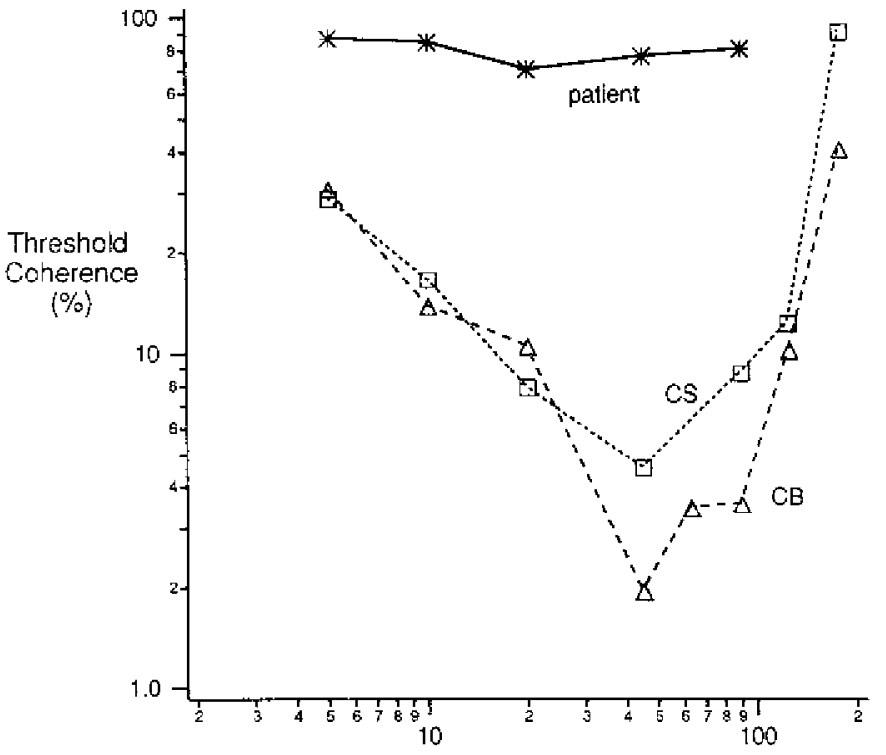

ISI (msec)

Figure 3. Same as Figure 2, but as function of ISI between successive reappearances of given dot, while keeping spatial displacement fixed at $4.3 \mathrm{~min}$ arc. Again, the patient was unable to achieve good performance at any value of ISI over the range $(40-100 \mathrm{msec})$ in which normal observers were able to obtain thresholds as low as $5 \%$.

every 45 msec; thus, they behaved like signal dots of only 1 -point lifetime.

Figure $4 A$ shows psychometric functions of direction discrimination performance as functions of stimulus coherence (percentage of signal dots) for a dot lifetime of 2 refreshes and a displacement of 19.4 min arc. Broken lines indicate data from 3 normal observers, who were generally able to discriminate reliably down to about $5 \%$ coherence. The performance of L.M., however, declined precipitously as even very small amounts of noise were added.

In an effort to obtain better performance from the patient, the displacement value was decreased to $4.3 \mathrm{~min}$ arc, which is closer to her optimum (Fig. 2). Data from this measurement are shown in Figure 4, $B$ and $C$, for dot lifetimes of 2 and 4 refreshes, respectively. The longer lifetime did yield somewhat better performance, both in the patient and in a normal observer, but in all these cases, L.M. still exhibited a profound deficit.

Over the range of coherence values in which L.M.'s performance declined, these stimuli gave an appearance (to normal observers) of 2 superimposed sets of signal and noise dots, which were effortlessly segregated into 2 transparent surfaces; the only salient change with decreasing coherence was a decline in signal dot density. We wondered whether the main effect here might be due to signal dot density rather than to the addition of noise, and therefore, we performed the following experiment.

\section{Invisible noise}

The above experiment was repeated, but with the noise dots plotted off the visible part of the display screen. Thus, a decrease of percent coherence had no effect other than to decrease the density, or number, of signal dots. The results, shown in Figure 5 , reveal dramatically better performance of the patient, down to very small percent coherences (i.e., densities). Thus, while L.M. does exhibit a deficit of performance for very low signal dot densities, this does not account for the sharp reduction of performance shown in the previous experiment.

\section{Lifetimes}

We next explored the effect of signal dot lifetime, keeping $100 \%$ coherence (i.e., no noise dots at all). To increase our temporal resolution in these measurements, the ISI was reduced to 10 msec. Psychometric functions were obtained of percent correct against dot lifetime and are shown in Figure $6, A$ and $B$, for 2 values of displacement. (The ratios of displacement to ISI correspond approximately to the 2 velocities of Fig. 4.) In general, the direction discrimination ability of both the patient and normal observers declined with decreasing dot lifetime. As in previous tests, L.M. exhibited poorer performance than the normal observers; however, the difference was considerably less marked,

A
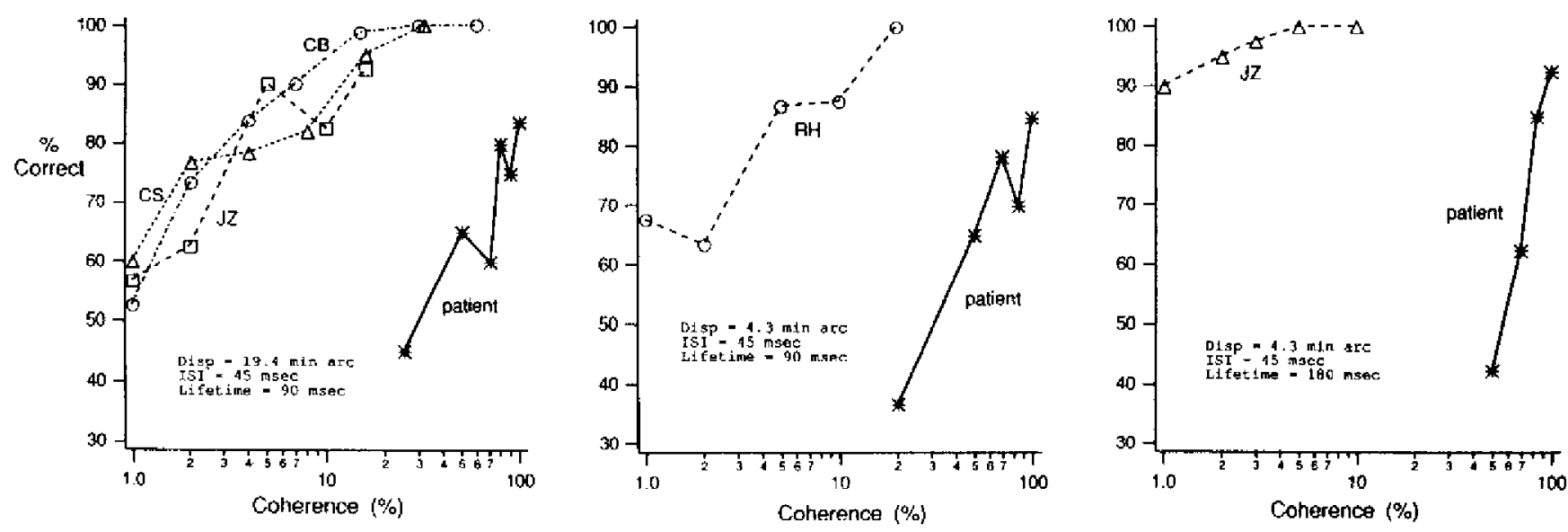

Figure 4. Psychometric functions of percentage correct direction discrimination, as functions of percentage coherence, using dot noise stimulus. $A$, Normal observers' performance declined gradually with decreasing coherence, with relatively good performance for coherence values as low as $10 \%$. The patient's performance, however, dropped precipitously with coherence, indicating an acute vulnerability to added noise dots. $B$ and $C$, Same as $A$, but for a smaller spatial displacement and longer dot lifetime. 


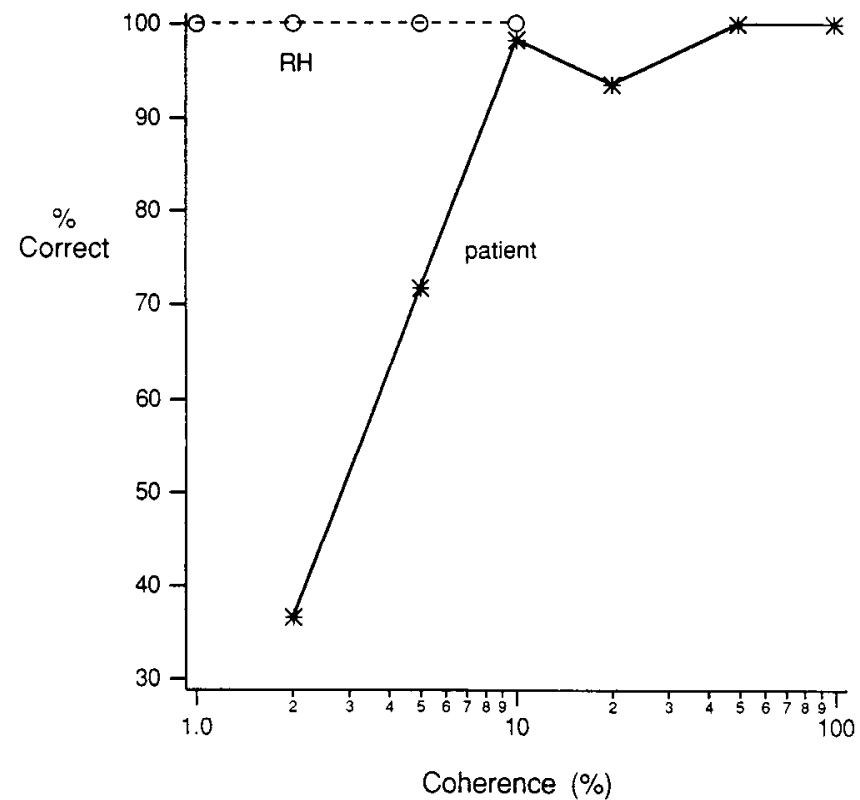

Figure 5. Psychometric function as in Figure 4, but using invisible noise stimulus. Because noise dots are not visible, decreasing values of coherence merely correspond to lower densities of signal dots. Note that, now, in the absence of noise, the patient's performance was greatly improved down to much lower coherence values. This indicates that the dramatic deficit revealed in the data of Figure 4 was due to the effect of added noise and not to the concomitant decline in signal dot density.

particularly in the case of the higher velocity (Fig. $6 A$ ). Also, unlike in other tcsts, we encountered some degree of dispersion of performance among the normal observers.

Thus, while the patient does show a deficit in terms of minimum required dot lifetime, this deficit is not in the quantitative range to account for the basic result of poor performance in the Movshon noise experiment (Figs. 1-3). For example, note in Figure 2 that, at $17.1 \mathrm{~min}$ arc displacement, the patient has a very impaired coherence threshold (above 90\%); at this ISI (45 $\mathrm{msec}$ ), most of the dot lifetimes will be well above $90 \mathrm{msec}$, in the range where the patient exhibits near errorless performance in the absence of noise (Fig. 6A).

Consequently, the Movshon noise result seems more likely to reflect primarily a deficiency in handling signal degradation by noise, rather than a problem with perception of short lifetimes.

\section{Immortal noise}

The above series of experiments, taken together, suggested that the patient's principal difficulty in perception of this class of stimuli resulted from the detrimental effects of additive noise. Because of the seemingly effortless perceptual segregation (in normal observers) of the dot noise stimuli into transparent planes of coherent signal dots and incoherent noise dots (over the range of coherence values that revealed a deficit), we wondered what other kinds of noise might act similarly. The noise dots of the dot noise experiment were of minimal possible lifetime (only 1 refresh), so we tested the other logical extreme: noise dots of indefinitely prolonged lifetime that were stationary. Again, it was the case that signal and noise dots were perceived (by normal observers) to be effortlessly segregated into transparent planes, in this case, one stationary and one moving. This task was so easy for normal observers that hardly any errors could be obtained.

\footnotetext{
'Note that a low coherence value for this stimulus results in a very small expected number of signal dots, whose motion is nevertheless easily perceived; some errors resulted from the actually plotted number of signal dots being 0 , on some trials.
}
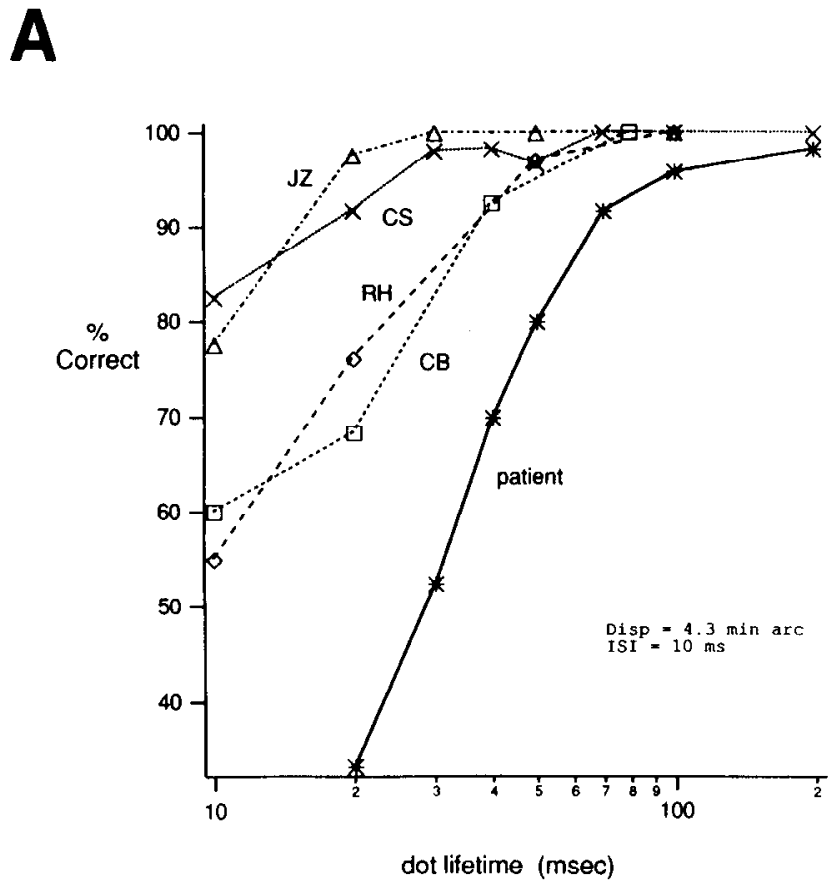

B

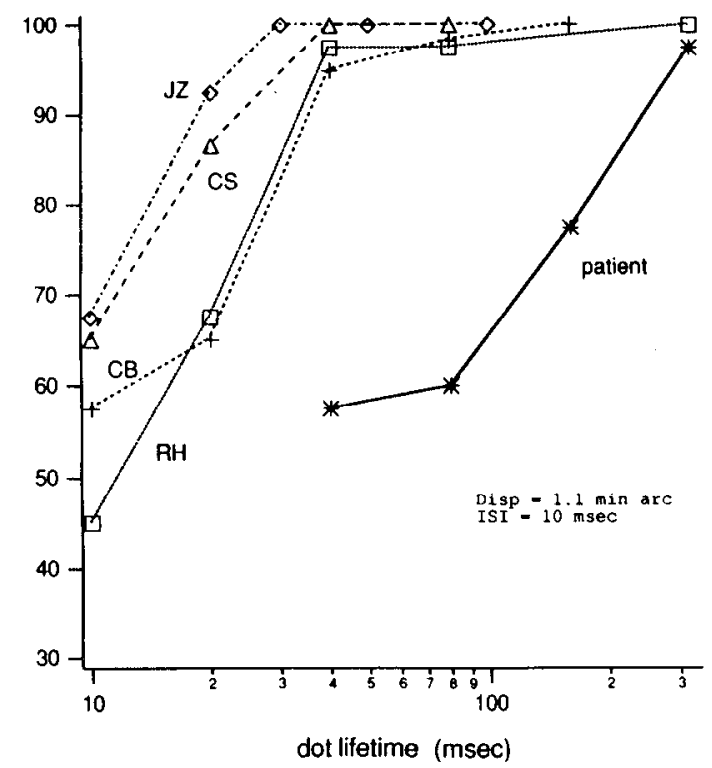

Figure 6. Effect of dot lifetime. Psychometric functions like those of Figure 4 are shown, in which the coherence was fixed at $100 \%$. Note the general decline in performance with decreasing dot lifetime, in both normal observers and, somewhat more rapidly, in the patient. $A$ and $B$ are for differing values of spatial displacement. Note that direction discrimination in the patient, compared to normal observers, is much more tolerant of reduced dot lifetime than of reduced signal-to-noise ratio (Fig. 4). 

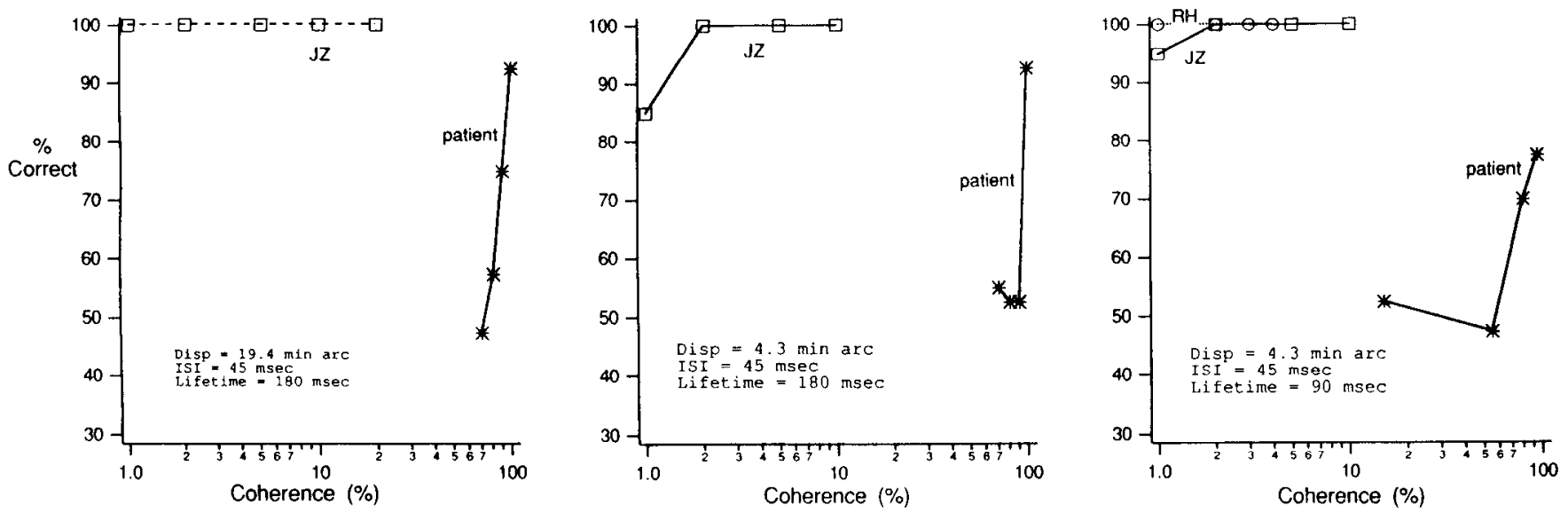

Figure 7. Effect of noise dots that do not move (immortal noise). Psychometric functions are shown for performance in an experiment like that of Figure 4, except that the noise dots are stationary. Normal observers were able to effortlessly segregate moving signal dots from stationary noise dots and discriminate direction almost flawlessly. The patient's performance, however, was devastated by even very small percentages of such stationary dots. $A-C$ demonstrate that the phenomenon was robust for differing stimulus parameter values.

However, L.M. exhibited an extraordinary deficit of performance on this task, which was robust for different dot lifetimes and displacements (Fig. 7A-C). Note that her performance had dramatically declined when only $5-10 \%$ of the dots were noise dots.

\section{Discussion}

Our measurements on the patient L.M. using the Movshon noise stimulus indicate a profound deficit of direction discrimination, which is quantitatively similar to that reported for acutely MTlesioned monkeys by Newsome and Pare (1988). This comparison is illustrated in Figure 8, which shows our data (from Fig. 2) and Newsome and Pare's data (their Fig. 9) for their complete unilateral MT-lesioned monkey (at $1 \mathrm{~d}$ postlesion). One of the major differences between this patient and the MT-lesioned monkeys of Newsome and Pare (1988) is in the degree of recovery. The monkeys showed substantial recovery of motion detection within a matter of days, possibly duc in some way to the rather small size of the unilateral excitotoxic acid lesions. L.M., however, suffered loss of a much larger, bilateral region of cortex (Zihl et al., 1991), where such recovery evidently does not occur.

The patient was essentially only able to perform the Movshon noise task for near-unity values of coherence, that is, smoothly drifting random dot patterns. Further experiments with other types of stochastic stimuli (dot noise, lifetimes) were used to separately assess the contributions of noise, as opposed to limited dot lifetime, in causing this deficit; our results indicate a much greater contribution to the deficit from difficulty in discrimination of signal in the presence of noise.

The patient's good performance at relatively short lifetimes (Fig. 6) makes it seem unlikely that her residual ability could be mediated by attentive tracking of particular dots or dot clusters. These data also indicate that any possible specific deficit of spatiotemporal "recruitment" (Nakayama and Silverman, 1984; Snowden and Braddick, 1989, 1990), if present, is not in itself sufficient to account for the severity of loss in the Movshon noise task.
Because our lifetimes experiment was performed with a 10msec temporal resolution, it remains logically conccivablc that L.M.'s deficit on the Movshon noise task might be due to an elevated minimal dot lifetime, expressed in number of views rather than in milliseconds (as we assumed). However, the precipitous rate at which her performance declined with coherence made this possibility seem a priori unlikely: at, for example, $80 \%$ coherence (below the patient's threshold), a substantial fraction of the dots still enjoy a rather long lifetime. While we did not perform a separate experiment to explicitly address this question, it seems unlikely in view of the patient's quite good performance on certain key conditions in other experiments. For example, consider the $100 \%$ coherence condition in the dot noise experiment (Fig. 4B); a 90-msec lifetime corresponds to only 3 views, yet the patient achieves more than $80 \%$ correct. In the comparable lifetime experiment (Fig. $6 A$ ), 3 views corresponds to a lifetime of $20 \mathrm{msec}$, at which the patient is at essentially chance performance. If the patient's fundamental deficit were an inability to process motion signals with very few views, her performance on the dot noise task at $100 \%$ coherence should have been equally poor.

Mikami et al. (1986) found that MT neurons respond to higher velocities and larger spatial displacements in apparent motion than striate cortex neurons. Thus, if L.M.'s lesion did involve area MT, one might have expected her to have greater loss of motion perception for larger values of spatial displacement, and possibly also at lower values of ISI; but, in fact, the data of Figures 2 and 3 show that L.M.'s best residual performance was at about the same spatial and temporal displacements as in the normal (2-20 min arc and 10-100 msec, respectively). It might be noted that this best set of parameters corresponds to a somewhat lower optimal velocity than that seen by Newsome and Pare (1988) in the monkey, or by Downing and Movshon (1989) in the normal human, probably because their stimuli were confined parafoveally rather than being centrally presented. The absolute threshold coherence values were also slightly higher in Downing and Movshon's (1989) data than in our normal data; this difference was probably due to the level of stimulus uncer- 

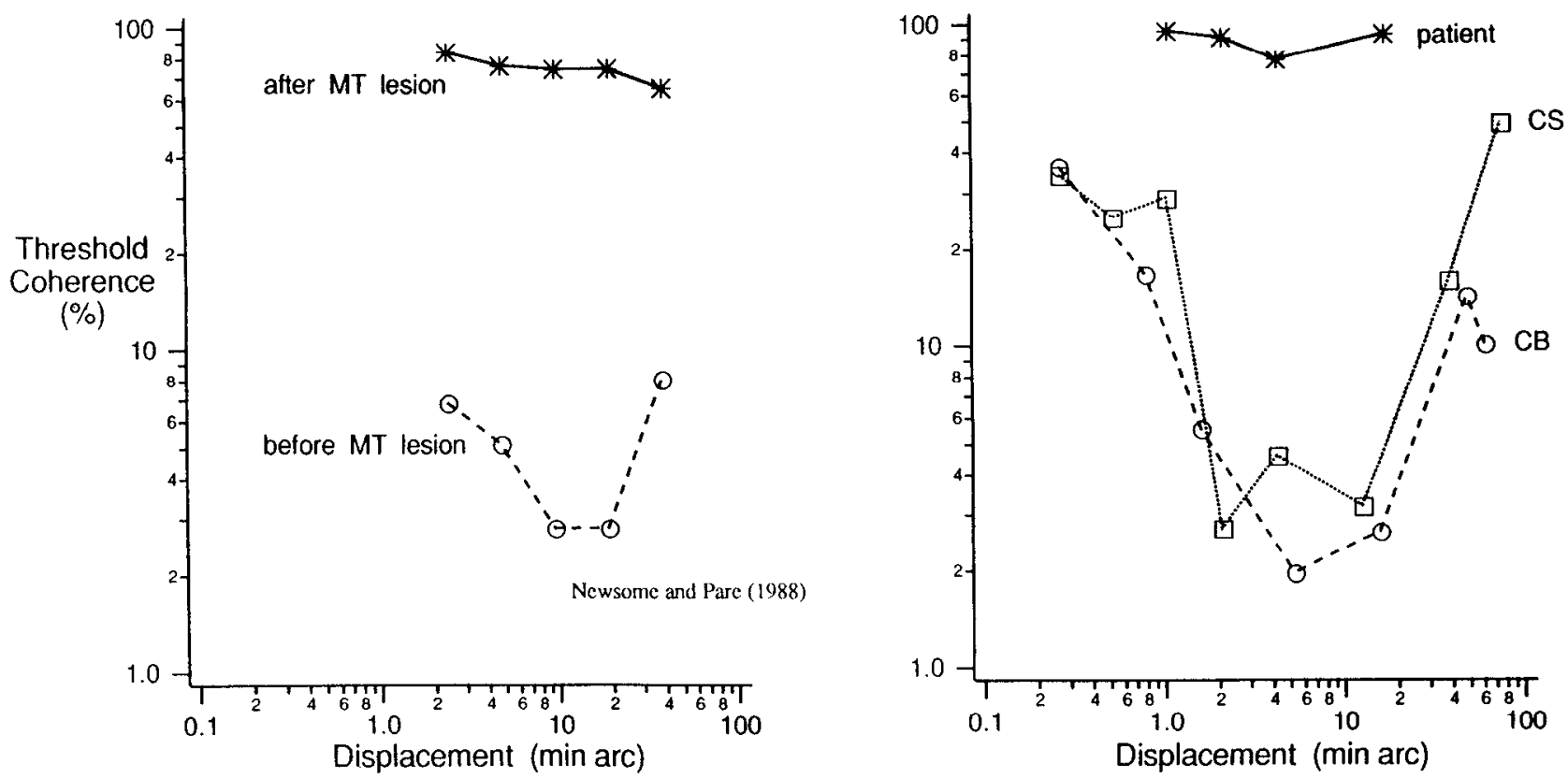

Figure 8. Comparison of patient's performance with that of MT-lesioned monkey. A, Threshold coherence values for the Movshon noise stimulus as a function of spatial displacement, replotted from published data (Newsome and Pare, 1988, their Fig. 9), for acutely MT-lesioned monkey; the broken line and open symbols indicate prelesion performance, while the solid line and solid symbols are for $1 \mathrm{~d}$ postlesion. B, Same as $A$, but for patient L.M. (replotted from Fig. 2). Note the similarity in overall form of the data, except for a quantitative shift of best performance to larger values of displacement in $A$; this difference may be due to the greater retinal eccentricity of stimulus placement used by Newsome and Pare (1988).

tainty in their task (their observers were required to identify correctly the location as well as the direction of motion).

This range of best spatial and temporal values corresponds to those of the "short-range process" of apparent motion (Braddick, 1974; Baker and Braddick, 1985a,b). Thus, the deficit involves the short-range mechanism (though these data do not rule out a deficit in the long-range process, also). On the other hand, by the same reasoning, the patient's residual direction discrimination performance at very high coherence values may be interpreted as a remaining degree of short-range competence.

Recently, other instances of selective loss of certain aspects of motion perception in brain-damaged patients have been reported (Vaina, 1989; Vaina et al., 1989a,b). At present, it is difficult to assess the comparability of these patients' lesions with that of the patient L.M. described here. However, it is noteworthy that thesc individuals suffered loss of extrastriate cortical areas in at least roughly similar regions, and also exhibited selective loss of certain types of motion perception. Recent studies (L.M. Vaina, personal communication) of such individuals using stochastic stimuli of the type used by Newsome and Pare (1988) also demonstrated profound deficits.

A rather different kind of motion blindness is seen in animals that have been reared in a stroboscopically illuminated environment; the visual cortex of such animals is lacking in directionally selective neurons (Cynader and Chernenko, 1976). These animals also show psychophysical deficits of motion perception, comparable to those of the patient described here: poor contrast sensitivity for discrimination of drift direction of sine-wave gratings (Pasternak et al., 1985) and large deficits for direction dis- crimination of stochastic dot stimuli (Harvitt et al., 1989) of the type described by Williams and Sekuler (1984).

Limited-lifetime random stimuli such as those used here hold great promise as an important tool for psychophysical exploration of both clinical and normal vision, as well as for single unit neurophysiology. One of their advantages is the possibility of many different ways to design stochastic stimuli to address differing questions, for example, the separation of noise versus lifetime factors in the experiments reported here. In addition, there is some evidence that these stimuli may provide a means of assessing psychophysical performance that is limited by neurons in extrastriate visual cortical areas, possibly area MT and/ or associated areas of the parietal "motion" pathway: Downing and Movshon (1989) demonstrated spatial summation effects and eccentricity dependence quantitatively consistent with known characteristics of these extrastriate areas.

The most surprising of these results is the severe impairment of motion perception in the presence of noise dots that are not even moving (the immortal noise experiment). The noise dots here might be thought of as effectively reducing the relative contrast of the signal dots; however, the patient's nearly normal contrast sensitivity (Hess et al., 1989) makes an explanation in these terms seem unlikely. In view of the considerably smaller degree of impairment found in the lifetimes experiment, the immortal noise result indicates that the patient's deficit cannot be simply characterized in terms of expected number of dot signal pairs, as described by Downing and Movshon (1989) for normal motion perception.

Another curious result is the patient's deficit at very low signal 
dot densities, even in the absence of noise (the invisible noise experiment; Fig. 5). This result is reminiscent of our previous demonstration of a severe deficit for direction discrimination of sine-wave gratings at low contrast (Hess et al., 1989, their Fig. 12). Both cases involve no experimentally introduced noise but, in differing ways, involve a reduced signal. Taken together with the vulnerability to noise shown here, these results suggest that the patient's loss might, in general, be better thought of in terms of an impairment in processing stimuli with a low signalto-noise ratio. An intriguing idea is that such a loss might arise simply from a reduction in number of neurons that carry directional information, rather than from an all-or-none loss of a discrete brain area or brain function.

In summary, the findings reported here suggest that one major role of extrastriate cortical processing might be the interpretation of stimuli that suffer from an impoverished signal-to-noise ratio; the most commonly encountered form of noise would presumably be contamination by irrelevant directional spatiotemporal frequency components.

\section{References}

Allman JM, Kaas JH (1971) A representation of the visual field in the caudal third of the middle temporal gyrus of the owl monkey (Aotus trivirgatus). Brain Res 31:85-105.

Baker CL Jr, Braddick OJ (1985a) Eccentricity-dependent scaling of the limits for short-range apparent motion perception. Vision Res 25: 803-812.

Baker CL Jr, Braddick OJ (1985b) Temporal properties of the shortrange process in apparent motion. Perception 14:181-192.

Braddick OJ (1974) A short-range process in apparent motion. Vision Res 14:519-527.

Cynader MS, Chernenko G (1976) Abolition of direction selectivity in the visual cortex of the cat. Science 193:504-505.

Downing CJ, Movshon JA (1989) Spatial and temporal summation in the detection of motion in stochastic random dot displays. Invest Ophthalmol Vis Sci [Suppl] 30:72.

Dubner R, Zeki SM (1971) Response properties and receptive fields of cells in an anatomically defined region of the superior temporal sulcus. Brain Res 35:528-532.

Harvitt D, Albano J, Pasternak T (1989) The role of cortical directional selectivity in perception of coherent motion. Soc Neurosci Abstr 15: 53.11.

Hess RF, Baker CL Jr, Zihl J (1989) The "motion-blind" patient: lowlevel spatial and temporal filters. J Neurosci 9:1628-1640.

Maunsell JHR, Newsome WT (1987) Visual processing in monkey extrastriate cortex. Annu Rev Neurosci 10:363-401.
Mikami A, Newsome W1, Wurtz RH (1986) Motion selectivity in macaque visual cortex. II. Spatiotemporal range of directional interactions in MT and V1. J Neurophysiol 55:1328-1339.

Morgan MJ, Ward R (1980) Conditions for motion flow in dynamic visual noise. Vision Res 20:431-435.

Nakayama K, Silverman GH (1984) Temporal and spatial characteristics of the upper displacement limit for motion in random dots. Vision Res 24:293-299.

Newsome WT, Pare EB (1988) A selective impairment of motion perception following lesions of the middle temporal visual area (MT). J Neurosci 8:2201-2211.

Pasternak T, Schumer RA, Gizzi MS, Movshon JA (1985) Abolition of visual cortical direction selectivity affects visual behavior in cats. Exp Brain Res 61:214-217.

Siegel RM, Andersen RA (1988) Perception of three-dimensional structure from motion in monkey and man. Nature 331:259-261.

Snowden RJ, Braddick OJ (1989) Extension of displacement limits in multiple-exposure sequences of apparent motion. Vision Res 29:17771787.

Snowden RJ, Braddick OJ (1990) Differences in the processing of short-range apparent motion at small and large displacements. Vision Res 30:1211-1227.

Vaina LM, LeMay M, Tootell R, Bienfang DC (1989a) Functional streams in human visual cortex: clinical evidence for a segregation of color and motion processing. Invest Ophthalmol Vis Sci [Suppl] 30: 409.

Vaina LM, LeMay M, Choi A, Kemper T, Bienfang D (1989b) Visual motion analysis with impaired speed perception: psychophysical and anatomical studies in humans. Soc Neurosci Abstr 15:497.8

van Essen DC (1985) Functional organization of primate visual cortex. In: Cerebral cortex (Peters A, Jones EG, eds), pp 259-329. New York: Plenum.

van Santen JPH, Sperling G (1985) Elaborated Reichardt detectors. J Opt Soc Am A 2:300-321.

Weibull WA (1951) A statistical distribution function of wide applicability. J Appl Mech 18:292-297.

Williams DW, Sekuler R (1984) Coherent global motion percepts from stochastic local motions. Vision Res 24:55-62.

Zeki SM (1974) Functional organization of a visual area in the posterior bank of the superior temporal sulcus of the rhesus monkey. $J$ Physiol (Lond) 236:549-573.

Zihl J, von Cramon D, Mai N (1983) Selective disturbance of movement vision after bilateral brain damage. Brain 106:313-340.

Zihl J, von Cramon D, Mai N, Schmid C (1991) Disturbance of movement vision after bilateral posterior brain damage: further evidence and follow-up observations. Brain, in press.

Zucker SW (1984) Type I and type II processes in early orientation sclcction. In: Figural synthesis (Dodwell PC, Caelli T, eds), pp 283 300. London: Lawrence Erlbaum. 